\title{
Fast Generation of Near-Optimal Plans for Eco-Efficient Stowage of Large Container Vessels
}

Pacino, Dario; Delgado, Alberto; Jensen, Rune Møller; Bebbington, Tom

Published in:

Proceedings of the International Joint Conference on Artificial Intelligence

Publication date:

2011

Document Version

Peer reviewed version

Link back to DTU Orbit

Citation (APA):

Pacino, D., Delgado, A., Jensen, R. M., \& Bebbington, T. (2011). Fast Generation of Near-Optimal Plans for Eco-Efficient Stowage of Large Container Vessels. Proceedings of the International Joint Conference on Artificial Intelligence, 286-301.

\section{General rights}

Copyright and moral rights for the publications made accessible in the public portal are retained by the authors and/or other copyright owners and it is a condition of accessing publications that users recognise and abide by the legal requirements associated with these rights.

- Users may download and print one copy of any publication from the public portal for the purpose of private study or research.

- You may not further distribute the material or use it for any profit-making activity or commercial gain

- You may freely distribute the URL identifying the publication in the public portal 


\title{
Fast Generation of Near-Optimal Plans for Eco-Efficient Stowage of Large Container Vessels
}

\author{
Dario Pacino ${ }^{1}$, Alberto Delgado ${ }^{1}$, Rune Møller Jensen ${ }^{1}$, and Tom Bebbington ${ }^{2}$ \\ 1 IT-University of Copenhagen, Denmark \{dpacino, alde, rmj\}@itu.dk \\ 2 Maersk Line Operations, Global Stowage Production, Singapore \\ Tom.Bebbington@maersk.com
}

\begin{abstract}
Eco-Efficient stowage plans that are both competitive and sustainable have become a priority for the shipping industry. Stowage planning is NP-hard and is a challenging optimization problem in practice. We propose a new 2-phase approach that generates near-optimal stowage plans and fulfills industrial time and quality requirements. Our approach combines an integer programming model for assigning groups of containers to storage areas of the vessel over multiple ports, and a constraint programming and local search procedure for stowing individual containers.
\end{abstract}

\section{Introduction}

Cost-efficiency and sustainability are not opposed objectives for the stowage plans generated daily by liner shipping companies. A stowage plan assigns containers to load in a port to vessel slots. An eco-efficient stowage plan aims at achieving a minimum port stay. In this way, port fees are minimized and the vessel maximizes its time at sea, which can be used to reduce its speed and thus bunker costs and $\mathrm{CO}_{2}$ emissions.

Eco-efficient stowage plans, however, are hard to produce in practice. First, they are made under time pressure by human stowage coordinators just hours before the vessel calls the port. Second, deep-sea vessels are large and often require thousands of container moves in a port. Third, complex interactions between lowlevel stacking rules and high-level stress limits and stability requirements make it difficult to minimize the makespan of cranes and, at the same time, avoid that containers block each other (overstowage). Finally, according to our industrial partner, stowage planning optimization algorithms must be fast. Runtimes of more than 10 minutes are impractical, since stowage coordinators possibly need to run several forecast scenarios.

This paper introduces a new stowage planning optimization approach that, similar to the currently most successful approaches (e.g, $[26,18,1])$, decomposes
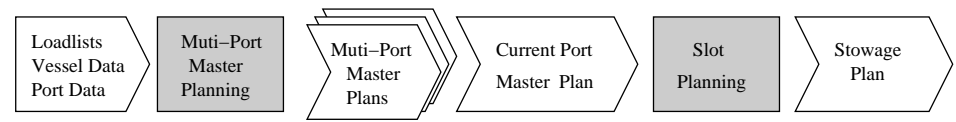

Fig. 1. Hierarchical decomposition of stowage planning into master and slot planning. 
the problem hierarchically as depicted in Figure 1. First the multi-port master planning phase decides how many containers of each type to stow in a set of storage areas of the vessel using an Integer Programming (IP) model. Based on this distribution, a complete stowage plan is generated in the Slot Planning phase where individual containers are stowed by a combination of Constraint Programming (CP) and Local Search (LS) models implemented in [8, 21]. To avoid negative impact of the final stowage plan in future ports, the multi-port master planning phase optimizes simultaneously master plans for the current port and a number of downstream ports.

We evaluated our approach experimentally on 20 real instances provided by our industrial partner. Despite the NP-hardness of multi-port master planning and the previous lack of success solving the problem optimally for large vessels over multiple ports (e.g., $[26,18,1]), 11$ of the 20 instances can be solved optimally in less than 10 minutes using a standard IP solver. More interestingly, the computation time can often be reduced several orders of magnitude if we relax the problem by dropping the integrality constraint on the decision variables in a mixed integer programming (MIP) model, without affecting solution quality.

A 2-phase approach for stowage planning, combining our MIP approach for multi-port master planning with our slot planning algorithms, can generate complete stowage plans in less than 330 seconds for 16 of the 20 instances. This runtime is well within the time bound of 10 minutes assessed by our industrial partner to be necessary for supporting stowage coordination in practice.

The remainder of the paper is organized as follows. Section 2 describes the problem. Section 3 introduces related work. Section 4 presents our 2-phase approach. Section 5 and 6 present the experiments and draw conclusions.

\section{Background and Problem Statement}

ISO containers transported on container ships are normally 8' wide, 8'6" high, and either 20', 40', or 45' long. Due to the lack of support points, it is not possible to stack a 20' container on a 40' or 45' container. High cube containers are 9'6" high and pallet wide containers are slightly wider and can only be placed side-by-side in certain patterns. Refrigerated containers (reefers) must be placed near power plugs. Containers with dangerous goods (IMO containers) must be placed according to a complex set of separation rules.

The capacity of a container ship is given in Twenty-foot Equivalent Units (TEUs). As shown in Figure 2, the cargo space of a vessel is divided into sections called bays and each bay is divided into an on deck and a below deck part by a number of hatch-covers, which are flat, leak-proof structures. A container is overstowing and causing extra crane moves if it is discharged later than a container stowed below it, either in the same stack or under a hatch-cover (hatchoverstowage). Each sub-section of a bay consists of a row of container stacks divided into slots that can hold a 20' ISO container. Figure 3 (a) and (b) show the container slots of a bay and stack, respectively. Stacks have max height and weight limits. Below deck, cell guides secure containers transversely. Containers 
on deck are secured by lashing rods and twist locks with limited strength. Thus, container weights must normally decrease upwards in stacks on deck. Moreover, lashing rods of 20' stacks must be accessible and stack heights must be under the vessel's minimum line of sight. 45' containers can normally only be stowed over the lashing bridge on deck.

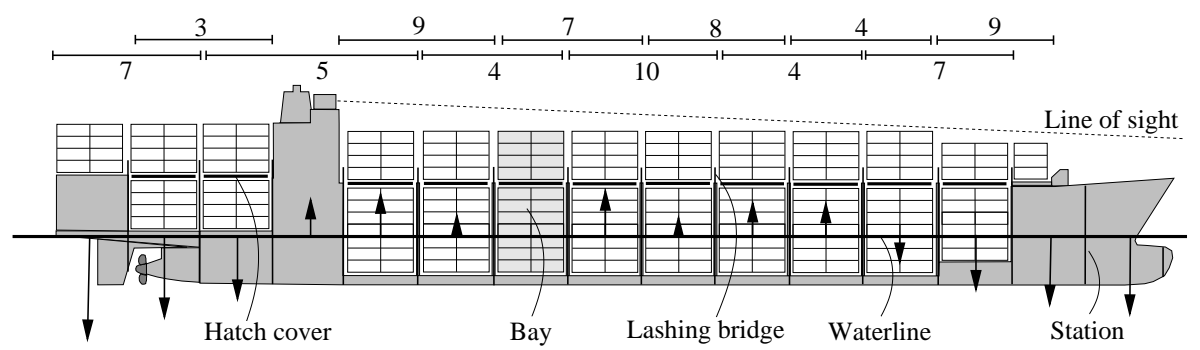

Fig. 2. The arrangement of bays in a small container vessel. The vertical arrows show an example of the resulting forces acting on the ship sections between calculation points (stations). Single crane work hours for adjacent bays are shown at the top.

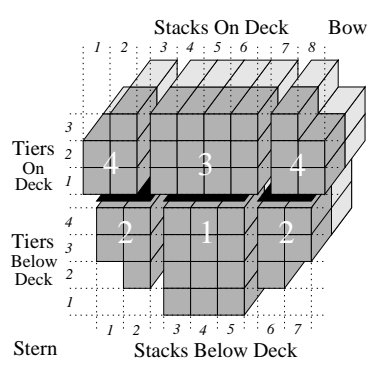

(a)

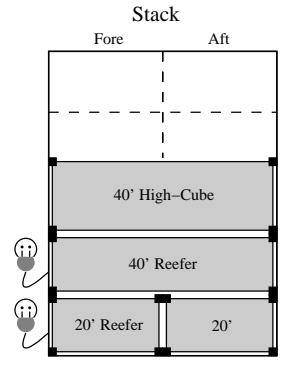

(b)

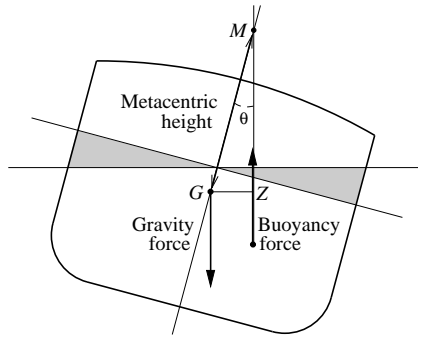

(c)

Fig. 3. (a) A vessel bay seen from behind. (b) A side view of a stack of containers. As depicted, power plugs are normally situated at bottom slots. (c) Transverse stability.

A container ship must sail at even keel and have sufficient transverse stability. Figure 3(c) shows a cross section of a ship. For small inclination angles, the volume of the emerged and immersed water wedges (shaded areas) and thus the distance $G Z$ are approximately proportional with the angle such that the buoyancy force intersects the center line in a fixed position called the metacenter, $M$ [24]. For an inclination angle $\theta$, the ship's uprighting force is proportional to $G Z=G M \sin \theta . G M$ is called the metacentric height and the center of gravity $G$ must be on the center line and result in sufficient $G M$ for the ship to be stable. Maximum and minimum draft restrictions apply due to port depths, working height of cranes, and the propeller. The trim is the difference between the aft and fore draft and must be kept within a given span. For a station position $p$, the shear force is the sum of the resulting vertical forces on vessel sections (see Figure 2) acting aft of $p$, and the bending moment is the sum of these forces 
times the horizontal distance to them from $p$. Both of these stresses must be within limits. The vessel also has transverse bending moment (torsion) limits. The meta center, draft, trim, and the buoyancy of each section of the vessel can be derived from its hydrostatic tables given its displacement and longitudinal center of gravity.

A container ship transports containers between ports on a fixed cyclic route. It is the liner shippers and not the port terminals that are in charge of producing stowage plans. A stowage plan assigns the containers to load in a terminal to slots on the vessel and it is often sent to the terminal shortly before calling it. The terminal guarantees a certain productivity in terms of the number and efficiency of assigned quay cranes, but otherwise the liner shipper has no control over its operations. Typically the terminal substitutes detailed container information for container types in order to optimize the load sequence [16]. In this work we assume the role of the liner shipper and focus on the generation of typed stowage plans for the current port. The plan is then completed by the terminal during the load sequencing, where the types are substituted with concrete containers.

The main objective of stowage planning is to minimize the port stay by reducing the total number of moves through overstowage minimization and distributing these moves evenly over the available quay cranes. Since quay cranes are too wide to work on two adjacent bays, a good lower bound for the makespan of quay cranes is the maximum work time of a single crane over pairs of adjacent bays (10 hours in the example shown in Figure 2). Stowage planning should take cargo in future ports into account (e.g., to ensure enough reefer capacity). But since cargo forecasts are inaccurate, the plan must be robust. Achieving this includes arranging containers with same port of discharge (POD) in vertical stacks rather than horizontal layers, freeing up as much bottom space as possible for unexpected reefer and long-haul containers, and to avoid mixing containers with different POD under deck to minimize the risk of hatch-overstows.

It is impractical to study large optimization models that include all details of stowage planning. On the other hand, all major aspects of the problem must be modeled for the results to be valuable. For container types this includes 20', 40', and reefer containers because short containers cannot be stowed over long ones which can force overstowage, and reefer slots are scarce and placed at the bottom which may force understowage. In addition, since stability, trim, draft

and stress moment limits should not fully be ignored, some weight classes of containers must be introduced. It is also important to take containers onboard the vessel when arriving the current port and containers to load in future ports into account. Finally since port stay minimization is essential, a realistic estimate of quay crane makespan and overstowage must be included.

\section{$3 \quad$ Literature Survey}

The number of publications on stowage planning has grown substantially within the last few years. Contributions can be divided into two main categories: singlephase and multi-phase approaches. Multi-phase approaches decompose the prob- 
lem hierarchically. They are currently the most successful in terms of model accuracy and scalability. The earliest work can be traced back to a 3-phase heuristic [25], but the first contribution that models several major aspects of the problem is a 2-phase approach that solves a master planning phase for multiple ports with a branch-and-bound algorithm and uses tabu search for bay planning [26]. Other approaches that use a similar decomposition include solving multi-port master planning with an iterative improvement approach based on the transportation simplex method [18] and a bin-packing heuristic [28]. 3-phase approaches include combinations of constructive heuristics, 0/1 IP, and metaheuristics (e.g., [1]) and heuristics combined with LS (e.g., [27]). Multi-phase approaches developed by the industry include a multi-stage placement heuristic using a number of lower-bounds [15] and a combination of sequential LP for master planning and a hierarchy of IPs for slot planning [14]. Except this last deployed industrial system which provides input data to our experiments, none of the previous approaches include all the major aspects of stowage planning mentioned at the end of Section 2.

Single-phase approaches represent the stowage planning problem (or parts of it) in a monolithic optimization model. The earliest work can be traced back to Aslidis $[4],{ }^{3}$ who makes a thorough study of overstow minimization algorithms for single bays. The remaining work is categorized according to solution approach. IP approaches include a very accurate but intractable model of the complete stowage problem [6] and simple models $[3,13,19]$ with limited scalability. $\mathrm{CP}$ approaches include an early simple model of the complete problem [2] and the combination of CP [8] and LS [21] used in this paper to generate near-optimal slot plans fast and robustly. A number of metaheuristics have been suggested: genetic algorithms (e.g., [7, 10]), simulated annealing [11], and placement heuristics (e.g., [5]). These approaches use simple models and often only focus on one aspect such as overstowage minimization. Additional single-phase approaches include simulation (e.g., [23]), expert systems (e.g., [9]), 3D-packing (e.g, [22]), and casebased methods (e.g., [20]). Also these approaches lack representative problem models.

\section{Solution Approach}

We propose the 2-phase approach shown in Figure 1. The first phase, multi-port master planning, distributes container types to sub-sections of bays for the entire route of the vessel. This phase deals with high-level constraints, modeling weight classes of standard 20' and 40' containers, and reefer containers. More precisely, we model GM stability, trim and draft limits, weight distribution and shear forces, and optimize hatch-overstowage and crane makespan. IMO, palletwide, and high-cube containers are not modeled. The two former are often stowed in specific areas, while the latter can be modeled with specialized capacity constraints. We also do not model bending moments and ballast water which are the focus of our future work. The input to muti-port master planning is industrial

\footnotetext{
${ }^{3}$ However, some unpublished work from the 1970s using simulation has been cited [6].
} 
data from a currently deployed stowage planning optimization tool [14], and it includes: 1) vessel data with ship layout and stress limits, 2) current port loadlist, and future port loadlists based on historical data, 3) port data with water depths, crane heights, and crane productivity. The input is adjusted such that for example weight and height constants fulfill quay crane height requirements and line of sight constraints. For that reason, the constants used in the models often change from port to port.

The master plan for the first port (the one we are making the stowage plan for) is used as input for the second phase, slot planning, to assign the containers of the types defined in the master plan to concrete slots. In slot planning, all major stacking rules apply: containers must form stacks, 20' containers cannot be stowed on top of 40' containers, reefer containers can only be stowed in reefer slots, stack maximum height and weight limits must be fulfilled and cell capacity must be observed. Containers are assigned with the aim of minimizing overstowage, clustering containers with the same POD and freeing stack and reefer slots for robustness.

\subsection{Multi-Port Master Planning}

The multi-port master planning phase assigns types of containers to sub-sections of bays (locations). Locations are either above or under a hatch-cover and are used as a tool to model hatch-overstowage. Figure 3(a) shows four locations within a bay. Outer locations are symmetrically split (such as location 2 and 4 in the Figure) to ease transverse stability calculations. For 20' and 40' containers, we consider a set of four mutually exclusive container types $T=\{L, H, R L, R H\}$, respectively light and heavy containers and light and heavy reefer containers. To produce a robust plan, our model takes into account the current and a set of downstream ports $P$. We define transports $T R$ as the set of pairs $\left\langle p_{1}, p_{2}\right\rangle$ where $p_{1}, p_{2} \in P$ are the loading and discharging port of a container type. We define two sets of decision variables $x_{t l}^{20 \tau}$ and $x_{t l}^{40 \tau}$ representing respectively the amount of 20' and 40' containers of type $\tau \in T$ to be stowed in location $l \in L$, where $L$ is the set of all locations, during transport $t \in T R$. Although the weight typing of containers used in the model might seem too simplistic, one has to take into account that the average weights, $W_{t}^{20 \tau}$ and $W_{t}^{40 \tau}$, for each type are calculated at transport level, making the classification much more refined. Following are the constraints of the proposed IP model:

$$
\begin{array}{lrl}
\sum_{t \in T R_{p}^{O N}} \sum_{\tau \in T}\left(x_{t l}^{20 \tau}+2 x_{t l}^{40 \tau}\right) \leq C_{p l}^{+} & \forall p \in P, l \in L \\
\sum_{t \in T R_{p}^{O N}} \sum_{\tau \in\{R L, R H\}}\left(x_{t l}^{20 \tau}+2 x_{t l}^{40 \tau}\right) \leq C_{p l}^{R} & \forall p \in P, l \in L \\
\sum_{t \in T R_{p}^{O N}} \sum_{\tau \in T} x_{t l}^{\alpha \tau} \leq C_{p l}^{\alpha} & \forall p \in P, l \in L, \alpha \in\{20,40\} \\
\sum_{l \in L} x_{t l}^{\alpha \tau}=L D_{t}^{\alpha \tau} & \forall \alpha \in\{20,40\}, \tau \in T, t \in T R
\end{array}
$$




$$
\begin{array}{cr}
\sum_{t \in T R_{p}^{O N}} \sum_{\tau \in T} \sum_{\alpha \in\{20,40\}} W_{t}^{\alpha \tau} x_{t l}^{\alpha \tau} \leq W_{p l}^{+} & \forall p \in P, l \in L \\
G_{p}^{-\rho} \leq \sum_{l \in L} G_{l}^{\rho} \sum_{t \in T R_{p}^{O N}} \sum_{\tau \in T} \sum_{\alpha \in\{20,40\}} W_{t}^{\alpha \tau} x_{t l}^{\alpha \tau} \leq G_{p}^{+\rho} & \forall p \in P, \rho \in\{L, V\} \\
S_{p s}^{-} \leq \sum_{l \in L_{s}^{A f t}} \sum_{t \in T R_{p}^{O N}} \sum_{\tau \in T} \sum_{\alpha \in\{20,40\}} W_{t}^{\alpha \tau} x_{t l}^{\alpha \tau} \leq S_{p s}^{+} & \forall p \in P, s \in S
\end{array}
$$

For each port $p \in P$, constraints (1) and (2) define the capacity restrictions of each location $l \in L$, respectively for the total number of TEUs allowed $\left(C_{p l}^{+}\right)$, and the number of reefer containers that can be stowed $\left(C_{p l}^{R}\right)$, where $T R_{p}^{O N}$ is the set of all the transports on the vessel at departure from port $p$. Similarly constraint (3) restricts the number of 20' $\left(C_{p l}^{20}\right)$ and 40' $\left(C_{p l}^{40}\right)$ that can be stowed in a location. Constraint (4) forces the loading of all containers in the loadlists. The amount of containers to load is given by the constants $L D_{t}^{20 \tau}$ and $L D_{t}^{40 \tau}$. The average weight of each container type under a specific transport $W_{t}^{\alpha \tau}$ is used in constraint (5) to limit the load of containers to the max weight allowance $W_{p l}^{+}$ reflecting draft limits and vessel capacity. Stability constraints can be calculated w.r.t. the center of gravity of the ship using the hydrostatic data table, and thus be satisfied by limiting its position using constraint (6). For each port $p \in P$, the center of gravity limits, $G_{p}^{+\rho}$ and $G_{p}^{-\rho}$, have been calculated, where $\rho \in\{L, V\}$ represent the longitudinal and vertical components reflecting trim, $G M$, and draft limits. Due to the symmetrical definition of outer locations, we assume cargo to be equally stowed on each side of the ship thus making irrelevant the calculations of the transverse component $\left(G_{l}^{\rho}\right.$ indicate the components of the center of gravity for each location $l \in L$ ). Given a set of stations $s \in S$ (calculation points as shown in Figure 2), constraint (7) calculates the downward force created by the cargo aft of each station $s$, where $L_{s}^{A f t}$ is the set of locations aft of station $s$, and $S_{p s}^{+}$and $S_{p s}^{-}$are the maximum and minimum shear limits at station $s \in S$ for port $p \in P$. Since the weight of cargo is constant, buoyancy is included in the calculations behind the constant limits.

In the master planning phase, overstowage minimization focuses on hatchoverstowage. This is modeled by a number of binary variables $\delta_{p l} \in\{0,1\}$, indicating the presence of containers to load or unload at port $p \in P$ under on deck locations $L^{O}$. This is accomplished with the following constraint

$$
\sum_{i \in L_{l}^{U}}\left(R_{p i}^{D}+\sum_{t \in T R_{p}^{A}} \sum_{\tau \in T}\left(x_{t i}^{20 \tau}+x_{t i}^{40 \tau}\right)\right) \leq M \delta_{p l} \quad \forall p \in P, l \in L^{O}
$$

where $L_{l}^{U}$ is the set of locations under $l \in L^{O}, T R_{p}^{A}$ is the set of transports that are either loaded or unloaded in port $p$, and $R_{p i}^{D}$ is the containers already on board the vessel when arriving at the first port (hereafter referred to as the release) that are discharge from location $i$ in port $p$. The indicator variable can 
now be used to calculate hatch-overstowage as follows:

$$
R_{p l}^{O V}+\sum_{t \in T R_{p}^{O V}} \sum_{\tau \in T}\left(x_{t l}^{20 \tau}+x_{t l}^{40 \tau}\right)-M\left(1-\delta_{p l}\right) \leq y_{p l}^{O} \quad \forall p \in P, l \in L^{O}
$$

Constraint (9) defines the cost variable $y_{p l}^{O}$ which, given the set of transports $T R_{p}^{O V}$ (and release containers $R_{p l}^{O V}$ ) that overstow containers to load or unload at port $p \in P$, counts the overstowing containers. In constraints (8-11) we make use of BigM constants $M$ tightened to the upper bounds of the constraints.

Due to the fact that slot planning constraints force 20' containers to be stowed under 40' ones, there is the possibility of forcing the introduction of overstowage within locations. To alleviate this problem, we estimate the potential overstowage between $20^{\prime}$ and 40' containers within each location.

$$
\begin{aligned}
R_{p l}^{D 20}+\sum_{t \in T R_{p}^{A}} \sum_{\tau \in T} x_{t l}^{20 \tau} \leq M \phi_{p l} \quad & \forall p \in P, l \in L \\
R_{p l}^{O V 40}+\sum_{t \in T R^{O V}} \sum_{\tau \in T} x_{t l}^{40 \tau}-M\left(1-\phi_{p l}\right) \leq y_{p l}^{P} \quad & \forall p \in P, l \in L
\end{aligned}
$$

Constraint (10) introduces a new set of Boolean variables $\phi_{p l}$ for each port $p \in P$ and location $l \in L$, indicating the presence of 20' containers to load or unload. These indicator variables define the cost variable $y_{p l}^{P}$ in constraint (11). This variable holds the number of potential overstows between 40' and 20' containers within a location. $R_{p l}^{D 20}$ and $R_{p l}^{O V 40}$ are the number of containers in the release discharged and potentially overstowing in port $p$, respectively.

Optimization of crane utilization is modeled as the minimization of the makespan of cranes. We calculate a lower bound of the makespan of cranes $y_{p}^{T}$ in port $p$ as demonstrated in Figure 2 with the following constraint:

$$
C^{T i m e} \sum_{t \in T R_{p}^{A}} \sum_{l \in L_{b}} \sum_{\tau \in T}\left(x_{t l}^{20 \tau}+2 x_{t l}^{40 \tau}\right) \leq y_{p}^{T} \quad \forall b \in B, p \in P
$$

where $B$ is the set of adjacent bays, $L_{b}$ is the locations of $b \in B$, and $C^{\text {Time }}$ is the average crane time needed to load or unload a container.

Capacity constraints over reefer containers do not ensure feasibility of a location in the slot planning phase due to stacking rules. The following constraint

$$
\sum_{t \in T R_{p}^{O N}} \sum_{\tau \in T} F_{p l}^{\tau}\left(x_{t l}^{20 \tau}+2 x_{t l}^{40 \tau}\right)-C_{p l}^{R} \leq y_{p l}^{R} \quad \forall p \in P, l \in L
$$

alleviate this issue by reducing the maximum capacity of reefer containers within a location by a proportional factor $F_{p l}^{\tau}$, where $F_{p l}^{\tau}=C_{p l}^{R} / C_{p l}^{+}$for all non-reefer containers and a factor 1 for all reefer containers. The reduction is then captured in the cost variable $y_{p l}^{R}$.

The model minimizes a weighted sum of the cost variables

$$
\sum_{p \in P} \sum_{l \in L}\left(\mathcal{C}^{O} y_{p l}^{O}+\mathcal{C}^{P} y_{p l}^{P}+\mathcal{C}^{R} y_{p l}^{R}\right)+\sum_{p \in P} \mathcal{C}^{T} y_{p}^{T}
$$


The weights $\left(\mathcal{C}^{O}, \mathcal{C}^{P}, \mathcal{C}^{R}, \mathcal{C}^{T}\right)$ have been derived from the deployed system of our industrial partner and thus reflect a refined adjustment to the economy of stowage planning and the preferences of stowage coordinators.

Complexity of Multi-Port Master Planning The Hatch Overstow Problem (HOP) is NP-complete [17]. This problem models the same hatch-overstow objective as multi-port master planning. Given the optimization version of the HOP, we can reduce it to the multi-port master planning problem by having only one crane and using the reefer capacities to disallow containers below deck. Thus, multi-port master planning is NP-hard.

\subsection{Slot Planning}

The master plan for the first port, which is the port to generate a stowage plan for, becomes an input to the slot planning phase. In this way information is passed in a top-down fashion from the master planning to the slot planning phase. There is currently no information flowing in the other direction. The input defines the number of containers of each type to stow in each location of the vessel, and by generating a slot plan for each location, a type-based stowage plan is created. Each slot plan is an independent sub-problem. It must decide which container type (if any container at all) to stow in each slot of the location. The container types here correspond to those defined in the multi-port master planning phase (Section 4.1) and must satisfy the constraints and optimize the objectives mentioned in Section 4 for slot planning. We direct the reader to [8] for a more detailed presentation of the model.

The slot planning phase is solved using a combination of the CP and LS algorithms described in $[8,21]$. The $\mathrm{CP}$ algorithm is initially run with a time limit of one second. ${ }^{4}$ Optimal or near-optimal solutions are often found within this time frame. In some cases, however, the $\mathrm{CP}$ approach is not able to find solutions. When this happens, the LS algorithm is run and the resulting solution is used. Such situations originate in two cases: 1) the problem is too complex for CP to be solved in one second (only few cases), and/or 2) the problem is infeasible. The latter case happens due to the abstraction used in the multi-port master planning phase. For instance, when the total weight of containers assigned to a location is within limits, but due to stack weight limits and the arrangement of already onboard containers it is not possible to stow all containers in the location. Since the loadlist is not strict, and removing a few containers from the locations has small impact on the stability of the vessel, the LS algorithm has been modified to roll out containers that cannot be stowed.

\section{Computational Results}

To evaluate our approach, we use 20 instances from a stowage planning optimization tool [14] deployed by our industrial partner. Table 1 gives an overview

${ }^{4}$ This corresponds to an upper bound of about 100 seconds for a typical large vessel when solving all the slot planning sub-problems sequentially. 


\begin{tabular}{|c|c|c|c|c|c|c|c|c|}
\hline \multicolumn{9}{|c|}{ Instances Characteristics } \\
\hline \multirow{2}{*}{ ID } & \multicolumn{2}{|l|}{ Vessel } & \multicolumn{4}{|c|}{ Route } & \multicolumn{2}{|c|}{ Encoding } \\
\hline & Cap. (TEU) & Loc. & Ports & Util (\%) & Weight (\%) & Moves & Transp. & Bools \\
\hline 1 & 7490 & $\overline{90}$ & 8 & 49 & 30 & 6162 & 17 & 250 \\
\hline 2 & 9618 & 100 & 4 & 82 & 56 & 5306 & 19 & 180 \\
\hline 3 & 4755 & 67 & 4 & 55 & 49 & 2276 & 18 & 118 \\
\hline 4 & 9160 & 100 & 16 & 87 & 54 & 9610 & 54 & 623 \\
\hline 5 & 7344 & 71 & 10 & 69 & 62 & 8736 & 76 & 618 \\
\hline 6 & 5044 & 76 & 14 & 81 & 76 & 8562 & 69 & 750 \\
\hline 7 & 6717 & 65 & 7 & 80 & 66 & 5462 & 56 & 238 \\
\hline 8 & 4478 & 36 & 9 & 78 & 59 & 1934 & 20 & 57 \\
\hline 9 & 5052 & 70 & 9 & 74 & 42 & 5272 & 83 & 577 \\
\hline 10 & 4755 & 67 & 5 & 70 & 72 & 3384 & 27 & 157 \\
\hline 11 & 9118 & 80 & 11 & 90 & 61 & 14290 & 109 & 850 \\
\hline 12 & 4456 & 61 & 6 & 41 & 35 & 4660 & 31 & 315 \\
\hline 13 & 4478 & 36 & 10 & 75 & 54 & 6790 & 81 & 352 \\
\hline 14 & 8490 & 100 & 6 & 81 & 58 & 6482 & 11 & 334 \\
\hline 15 & 5047 & 71 & 9 & 64 & 57 & 8208 & 88 & 585 \\
\hline 16 & 6545 & 80 & 6 & 10 & 9 & 834 & 10 & 234 \\
\hline 17 & 9984 & 87 & 4 & 69 & 33 & 3284 & 12 & 143 \\
\hline 18 & 2584 & 40 & 5 & 28 & 11 & 882 & 9 & 118 \\
\hline 19 & 9118 & 80 & 11 & 93 & 30 & 12182 & 25 & 728 \\
\hline 20 & 9160 & 100 & 10 & 79 & 62 & 9196 & 89 & 960 \\
\hline
\end{tabular}

Table 1. Problem instances overview. Columns under Vessel indicate ship dependent data: Cap. is the maximum nominal capacity of the ship and Loc is the total number of locations. Notice that given the same number of locations, different vessels can have different capacities. Columns under Route show information about the route, Ports indicates the number of calls in the route, Util. and Weight are the maximum utilization during the voyage in terms of TEU and weight. Moves is the total number of crane moves on the route. The Encoding columns present the number of Boolean variables (Bools) needed by the multi-port master planning phase after preprocessing, while Transp. is the total number of active transports.

of the characteristics of the instances. The instances are real stowage problems that coordinators have solved using the deployed tool and thus have very high data quality. All experiments were run on a Linux machine with two Six Core AMD Opteron processors at 2.0 Ghz and $32 \mathrm{~GB}$ of memory. Multi-Port master and slot planning models were implemented in $\mathrm{C}++$ and used respectively CPLEX 12.2, and Gecode 3.5 [12] libraries. Due to the non-deterministic nature of the LS algorithm, results of slot planning are reported in average over 10 runs of the algorithm.

\subsection{Multi-Port Master Planning Experiments}

Given that no previous approaches have solved the master planning problem to optimality, we did not expect our IP model to be efficient. The results, however, did rise attention. Table 2 presents the results of the IP model, and two experiments where the algorithm is stopped at a 2 and $5 \%$ gap from the LP relaxation. A $5 \%$ approximation is acceptable since forecasted loadlist data is imprecise. The results are quite interesting for two reasons. First, the IP model was able to calculate optimal solutions for 13 of the 20 instances within a 5 hours limit. Second, taking the results with a $5 \%$ gap, it is possible to generate a complete stowage plan within 10 minutes for 12 of the instances, suggesting 


\begin{tabular}{|c|c|c|c|c|c|c|c|}
\hline \multicolumn{8}{|c|}{ IP Results } \\
\hline \multirow[b]{2}{*}{ ID } & \multicolumn{2}{|c|}{ Optimal } & \multicolumn{2}{|c|}{$2 \%$ Gap } & \multicolumn{3}{|c|}{$5 \%$ Gap } \\
\hline & $\begin{array}{l}\text { Obj. } \\
\left(10^{5}\right)\end{array}$ & $\begin{array}{l}\text { Time } \\
\text { (sec.) }\end{array}$ & $\begin{array}{l}\text { Gap } \\
(\%)\end{array}$ & $\begin{array}{c}\text { Time } \\
\text { (sec.) }\end{array}$ & $\begin{array}{l}\text { Gap } \\
(\%)\end{array}$ & $\begin{array}{l}\text { Time } \\
\text { (sec.) }\end{array}$ & $\begin{array}{l}\text { Total } \\
\text { (sec.) }\end{array}$ \\
\hline 1 & 10.99 & 6.15 & 0.18 & 6.15 & 4.47 & 2.98 & 7.94 \\
\hline 2 & 18.73 & 7.41 & 1.30 & 2.52 & 1.30 & 2.52 & 16.15 \\
\hline 3 & 3.77 & 8.83 & 0.00 & 8.49 & 3.81 & 5.19 & 10.13 \\
\hline 4 & 28.87 & 385.68 & 0.14 & 229.08 & 3.63 & 96.65 & 110.28 \\
\hline 5 & 20.64 & 5988.48 & 2.11 & 1390.92 & 2.11 & 1390.92 & 1396.18 \\
\hline 6 & - & timeout & - & timeout & - & timeout & timeout \\
\hline 7 & 30.22 & 558.65 & 0.17 & 39.06 & 5.21 & 10.00 & 15.26 \\
\hline 8 & 77.52 & 2.24 & 1.31 & 1.12 & 1.31 & 1.12 & 5.2 \\
\hline 9 & 6.71 & 2476.37 & 0.11 & 1033.35 & 0.11 & 1033.35 & 1034.01 \\
\hline 10 & 6.85 & 19.30 & 0.01 & 16.38 & 4.29 & 6.90 & 17.95 \\
\hline 11 & - & timeout & - & timeout & - & timeout & timeout \\
\hline 12 & 2.89 & 24.71 & 0.00 & 7.06 & 0.00 & 7.06 & 15.01 \\
\hline 13 & - & timeout & - & 4658.59 & - & 2488.22 & 2497.41 \\
\hline 14 & 11.53 & 151.76 & 0.00 & 151.76 & 0.00 & 151.76 & 162.49 \\
\hline 15 & - & - & - & timeout & - & timeout & timeout \\
\hline 16 & 23.90 & 1.78 & 0.00 & 1.78 & 0.00 & 1.78 & 3.24 \\
\hline 17 & 4.62 & 1.32 & 0.00 & 1.32 & 0.00 & 1.32 & 15.30 \\
\hline 18 & - & timeout & - & 0.46 & - & 0.46 & 1.36 \\
\hline 19 & - & timeout & - & timeout & - & timeout & timeout \\
\hline 20 & - & timeout & - & 11319.07 & - & 7272.95 & 7282.80 \\
\hline
\end{tabular}

Table 2. Multi-Port Master Planning with IP. The first column is the instance number. The next columns present grouped results of three runs of the model: the first for optimality and the others ending respectively at 2 and $5 \%$ gap from the LP relaxation. Column Obj is the optimal value, and column Gap the distance to optimality w.r.t. Obj. Times are reported in Time, while time to generate a complete stowage plan is shown in column Total which includes the runtime of the slot planning phase. Instances that could not be solved within 5 hours are marked with timeout. The bold face shows results obtained within $10 \mathrm{~min}$.

the need for further research on IP models. As expected, the objective value is dominated by the overstowage objectives (9) and (11).

MIP relaxation To tackle the weaknesses of the IP model, we propose a mixed integer programming model where we relax the integrality constraints over the decision variables $x_{t l}^{20 \tau}$ and $x_{t l}^{40 \tau}$. We experimentally evaluate the MIP relaxation by comparing its results to the optimals from the IP model. Table 3 presents results for optimal runs and for 2 and $5 \%$ gap from the LP relaxation of the MIP model. In terms of objective value MIP and IP solutions are very similar. It is only in the $5 \%$ gap results that it is possible to notice some significant difference which, however, does not exceed $5.12 \%$. In contrast, runtime results are drastically different. It is now possible to generate complete stowage plans for nearly all instances within 10 minutes. Only 4 of the 20 instances could not be solved within this time frame, and 14 could be solved to optimality. Experiments have shown no difference in objective value w.r.t. overstowage. Also we see a very small difference in crane utilization, clearly due to the increased flexibility of the decision variables. This important observation can be used to advocate the use of the MIP model in exchange for IP. The IP model has to face the combinatorial problem of deciding whether one single container should be stowed in one location or another. Using a MIP we can split this container and avoid the combinatorial puzzle. Even a single container could, however, cause a large 


\begin{tabular}{|c|c|c|c|c|c|c|c|}
\hline \multicolumn{8}{|c|}{ MIP Results } \\
\hline \multirow[b]{2}{*}{ Inst. } & \multicolumn{2}{|c|}{ Optimal } & \multicolumn{2}{|c|}{$2 \%$ Gap } & \multicolumn{3}{|c|}{$5 \%$ Gap } \\
\hline & $\begin{array}{l}\text { Gap } \\
(\%)\end{array}$ & $\begin{array}{c}\text { Time } \\
\text { (sec.) }\end{array}$ & $\begin{array}{l}\text { Gap } \\
(\%)\end{array}$ & $\begin{array}{c}\text { Time } \\
\text { (sec.) }\end{array}$ & $\begin{array}{l}\text { Gap } \\
(\%)\end{array}$ & $\begin{array}{c}\text { Time } \\
\text { (sec.) }\end{array}$ & $\begin{array}{c}\text { Total } \\
\text { (sec.) }\end{array}$ \\
\hline 1 & $\overline{0.000}$ & 1.75 & $\overline{0.002}$ & 1.75 & 0.002 & 1.75 & 5.22 \\
\hline 2 & 0.000 & 3.57 & 0.007 & 2.38 & 0.007 & 2.38 & 15.59 \\
\hline 3 & 0.000 & 1.28 & 0.033 & 0.65 & 0.033 & 0.65 & 4.71 \\
\hline 4 & 0.004 & 272.90 & 1.647 & 84.27 & 2.478 & 35.04 & 51.48 \\
\hline 5 & 0.002 & 384.69 & 0.095 & 234.02 & 0.095 & 234.02 & 242.96 \\
\hline 6 & - & timeout & - & timeout & - & timeout & timeout \\
\hline 7 & 0.001 & 8.32 & 0.001 & 8.32 & 0.001 & 8.32 & 15.14 \\
\hline 8 & 0.009 & 0.97 & 0.295 & 0.67 & 0.295 & 0.67 & 2.18 \\
\hline 9 & 0.001 & 211.48 & 0.001 & 211.48 & 0.001 & 211.48 & 212.47 \\
\hline 10 & 0.004 & 7.15 & 0.133 & 4.52 & 3.545 & 1.24 & 10.43 \\
\hline 11 & - & timeout & - & 3708.52 & - & 3708.52 & 3709.47 \\
\hline 12 & 0.004 & 1.95 & 0.004 & 1.95 & 0.004 & 1.95 & 8.91 \\
\hline 13 & - & timeout & - & 311.33 & - & 311.33 & 321.01 \\
\hline 14 & 0.002 & 6.93 & 0.805 & 3.25 & 0.805 & 3.25 & 16.82 \\
\hline 15 & - & timeout & - & 251.42 & - & 251.42 & 256.63 \\
\hline 16 & 0.000 & 0.73 & 0.000 & 0.73 & 0.000 & 0.73 & 2.52 \\
\hline 17 & 0.005 & 0.67 & 1.744 & 0.52 & 1.744 & 0.52 & 10.25 \\
\hline 18 & - & 0.20 & - & 0.20 & - & 0.20 & 1.13 \\
\hline 19 & - & timeout & - & timeout & - & timeout & timeout \\
\hline 20 & - & timeout & - & 3636.18 & - & 2060.71 & 2070.46 \\
\hline
\end{tabular}

Table 3. Multi-Port Master Planning with MIP. The second column describes the gap between the IP and MIP optimal solution. For the other columns see Table 2.

amount of overstowage, but we do not see this happening in practice. We thus believe the MIP model can be used in practice by the industry. Using a MIP also gives the industry the ability to use standard solvers and eases the process of adding side constraints.

\subsection{Slot planning experiments}

The slot planning experiments discussed in this section are based on master plans obtained from the IP and MIP multi-port master planning experiment with 5\% optimality gap. Master plans based on MIP may have fractional numbers of containers to stow in locations which is physically impossible. We attempt to stow a fractioned container in one of the locations where a fraction of it has been assigned by the master plan. If none of these locations have capacity left, the container is rolled out.

Based on previous experiments $([8,21])$, we set up a time limit of one second for slot planning each location of a vessel. The quality of each slot plan is evaluated by comparing it with the best slot plan generated by CP for the same location within twenty minutes. In the cases where the number of containers of one or more types were reduced by LS, the slot plan is evaluated against the best plan generated by $\mathrm{CP}$ within twenty minutes, stowing the same containers as LS. Table 4 summarizes the results of our experiments.

Vessels are slot planned fast by our approach. For the instances with a master plan available, slot plans are generated within 17 seconds in total. There is no time-wise dominance of slot plans generated from MIP and IP master plans, indicating that integrality constraints do not affect the complexity of slot planning. When slot planning IP master plans, a reduction in the number of 


\begin{tabular}{|c|c|c|c|c|c|c|c|c|c|c|c|c|c|}
\hline \multirow{3}{*}{ ID } & \multicolumn{11}{|c|}{ Slot Planning Current Port } & & \\
\hline & \multirow{2}{*}{ Conts. } & \multicolumn{2}{|c|}{ Time(s) } & \multicolumn{2}{|c|}{ Locs. } & \multicolumn{2}{|c|}{ Frac.+Odd } & \multicolumn{2}{|c|}{ LS rolled } & \multicolumn{2}{|c|}{ Rolled out(\%) } & \multicolumn{2}{|c|}{$\operatorname{Gap}(\%)$} \\
\hline & & Cont. & Int. & Cont. & Int. & Cont. & Int. & Cont. & Int. & Cont. & Int. & Cont. & Int. \\
\hline 1 & 990 & 3.48 & 4.96 & 34 & 34 & 4 & 2 & 0 & 0 & 0.40 & 0.20 & 0.00 & 0.00 \\
\hline 2 & 2222 & 13.21 & 13.10 & 61 & 57 & 15 & 6 & 42.3 & 39.4 & 2.58 & 2.04 & 3.05 & 4.60 \\
\hline 3 & 783 & 4.07 & 4.94 & 33 & 32 & 3 & 7 & 0 & 0 & 0.38 & 0.89 & 0.36 & 0.00 \\
\hline 4 & 1876 & 16.44 & 13.64 & 69 & 70 & 14 & 5 & 19.1 & 15.2 & 1.76 & 1.08 & 12.77 & 10.47 \\
\hline 5 & 573 & 8.94 & 6.08 & 29 & 25 & 10 & 5 & 0 & 0 & 1.75 & 0.87 & 1.41 & 0.45 \\
\hline 6 & - & - & - & - & - & - & - & - & - & - & - & - & - \\
\hline 7 & 862 & 6.83 & 5.26 & 27 & 35 & 5 & 6 & 3 & 0 & 0.93 & 0.70 & 1.25 & 0.00 \\
\hline 8 & 238 & 1.52 & 4.08 & 12 & 14 & 1 & 1 & 0 & 0 & 0.42 & 0.42 & 0.00 & 0.00 \\
\hline 9 & 334 & 0.99 & 0.66 & 14 & 13 & 4 & 4 & 3 & 0 & 2.10 & 1.20 & 1.57 & 0.00 \\
\hline 10 & 1064 & 9.20 & 11.06 & 29 & 32 & 8 & 5 & 41.8 & 58.7 & 4.68 & 5.99 & 13.86 & 16.44 \\
\hline 11 & 314 & 0.95 & - & 12 & - & 1 & - & 0 & - & 0.32 & - & 0.00 & . \\
\hline 12 & 757 & 6.97 & 7.96 & 37 & 27 & 7 & 5 & 0 & 0 & 0.92 & 0.66 & 0.63 & 0.59 \\
\hline 13 & 894 & 9.69 & 9.19 & 24 & 23 & 0 & 2 & 0 & 0 & 0.00 & 0.22 & 0.44 & 0.67 \\
\hline 14 & 2190 & 13.57 & 10.73 & 61 & 58 & 7 & 5 & 21.5 & 0 & 1.30 & 0.23 & 0.78 & 0.03 \\
\hline 15 & 901 & 5.21 & - & 30 & - & 14 & - & 8.6 & - & 2.51 & - & 6.73 & - \\
\hline 16 & 302 & 1.79 & 1.46 & 16 & 14 & 2 & 6 & 0 & 0 & 0.66 & 1.99 & 0.00 & 0.00 \\
\hline 17 & 1618 & 9.73 & 13.98 & 50 & 50 & 4 & 6 & 5.8 & 41.6 & 0.61 & 2.94 & 0.38 & 1.54 \\
\hline 18 & 200 & 0.94 & 0.91 & 12 & 13 & 0 & 0 & 0 & 0 & 0.00 & 0.00 & 0.00 & 0.00 \\
\hline 19 & - & - & - & - & - & - & - & - & - & - & - & - & - \\
\hline 20 & 1261 & 9,82 & 7,73 & 38 & 37 & 6 & 4 & 0 & 1 & 0.48 & 0.40 & 2,01 & 9,75 \\
\hline
\end{tabular}

Table 4. Slot Planning MIP (Cont.) and IP (Int.) master plans with $5 \%$ gap. The first and second columns are the id of the instance and the number of containers to stow in the first port. The next columns show grouped results of slot planning based on MIP and IP master plans. The third and fourth columns show the runtime for the slot plans, fifth and sixth columns is the number of locations. The seventh and eighth columns totalize the number of rolled out containers by fractionality and odd number of 20' types, the ninth and tenth columns are the containers rolled out by LS, and eleventh and twelfth columns are the percentage of total containers rolled out. The last two columns show the average gap of the slot plans. A dash indicates that no master plan was provided.

containers rolled out due to fractionality and odd number of 20 ' types in locations is observed in most of the instances. This is, however, a very small $(0.4 \%$ $\max )$ fraction. The number of containers rolled out by LS differs in average only in 1.6 containers (9.7 for the IP and 8.1 for the MIP master plans), and the average percentage of total containers rolled out is the same $(1.2 \%)$ for both models. These facts indicate no considerable impact of using MIP master plans. Moreover, it was possible to generate slot plans for two extra instances using MIP master plans.

The maximum roll-out of an instance is $5.99 \%$, a reasonable number given the amount of containers typically rolled from a loadlist by stowage coordinators. Only two instances have an average optimality gap over $10 \%$, due to the presence of outliers, and the median of all instances is always $0 \%$. CP generated optimal slot plans within one second for $91.8 \%$ of the locations of the MIP master plans and $94.8 \%$ of the locations of the IP ones. Moreover, CP was able to prove optimality in $83 \%$ of the slot plans generated for the locations for both, the MIP and IP master plans. 


\section{Conclusion}

This paper presented a 2-phase stowage planning optimization approach able to solve industry instances to near-optimality within the time limits required for practical usage. In particular, we introduced an IP model of multi-port master planning that includes all major aspects of the problem. Our experiments have shown that multi-port master planning can be solved fast to optimality for a significant number of instances, and that runtime can be drastically reduced by relaxing integrality constraints without compromising solution quality. We have shown that in 16 of our 20 test instances, complete stowage plans can be computed in less than 330 seconds. The remaining four instances take longer due to the time needed for master planning. In future work we plan to develop heuristic master planning algorithms to handle such hard cases. We are also interested in optimizing ballast water and compare the performance of our system with human stowage coordinators.

Acknowledgments We would like to thank Stephen Barraclough, Jon Adam Freeman, Robert John Milton, and Mikkel Mühldorff Sigurd at Maersk Line and Nicolas Guilbert, Kim Hansen, Esben Mose Hansen, Benoit Paquin, Marc Cromme, and Hans Schou at Ange Optimization for their extensive support of this work. This research is sponsored in part by the Danish Maritime Fund under the BAYSTOW project.

\section{References}

1. Ambrosino, D., Anghinolfi, D., Paolucci, M., Sciomachen, A.: An experimental comparison of different heuristics for the master bay plan problem. In: Proceedings of the 9th Int. Symposium on Experimental Algorithms. pp. 314-325 (2010)

2. Ambrosino, D., Sciomachen, A.: A constraint satisfaction approach for master bay plans. Maritime Engineering and Ports 36, 175-184 (1998)

3. Ambrosino, D., Sciomachen, A.: Impact of yard organization on the master bay planning problem. Maritime Economics and Logistics (5), 285-300 (2003)

4. Aslidis, A.H.: Optimal Container Loading. Master's thesis, Massachusetts Institute of Technology (1984)

5. Avriel, M., Penn, M., Shpirer, N., Witteboon, S.: Stowage planning for container ships to reduce the number of shifts. Annals of Oper. Research 76, 55-71 (1998)

6. Botter, R., Brinati, M.A.: Stowage container planning: A model for getting an optimal solution. In: Proceedings of the 7th Int. Conf. on Computer Applications in the Automation of Shipyard Operation and Ship Design. pp. 217-229 (1992)

7. Davidor, Y., Avihail, M.: A method for determining a vessel stowage plan, Patent Publication WO9735266 (1996)

8. Delgado, A., Jensen, R.M., Schulte, C.: Generating optimal stowage plans for container vessel bays. In: Proceedings of the 15th Int. Conf. on Principles and Practice of Constraint Programming (CP-09). LNCS Series, vol. 5732, pp. 6-20 (2009)

9. Dillingham, J., Perakis, A.N.: Design of an expert system for container stowage planning. In: Proceedings of the Fleet Management Technology Conference. Baltimore (1987) 
10. Dubrovsky, O., Penn, G.L.M.: A genetic algorithm with a compact solution encoding for the container ship stowage problem. J. of Heuristics 8, 585-599 (2002)

11. Flor, M.: Heuristic Algorithms for Solving the Container Ship Stowage Problem. Master's thesis, Technion, Haifa, Isreal (1998)

12. Gecode Team: Gecode: Generic constraint development environment (2006), available from http://www.gecode.org

13. Giemesch, P., Jellinghaus, A.: Optimization models for the containership stowage problem. In: Proceedings of the Int. Conference of the German Operations Research Society (2003)

14. Guilbert, N., Paquin, B.: Container vessel stowage planning, Patent Publication US2010/0145501 (2010)

15. Gumus, M., Kaminsky, P., Tiemroth, E., Ayik, M.: A multi-stage decomposition heuristic for the container stowage problem. In: Proceedings of the $2008 \mathrm{MSOM}$ Conference (2008)

16. Imai, A., Sasaki, K., Nishimura, E., Papadimitriou, S.: Multi-objective simultaneous stowage and load planning for a container ship with container rehandle in yard stacks. European Journal of Operational Research (171), 373-389 (2006)

17. Jensen, R.M.: On the complexity of container stowage planning: the capacitated zero-shift problem and the hatch overstow problem. Tech. Rep. ITU-TR-137, IT University of Copenhagen (2010)

18. Kang, J., Kim, Y.: Stowage planning in maritime container transportation. Journal of the Operations Research Society 53(4), 415-426 (2002)

19. Li, F., Tian, C., Cao, R., Ding, W.: An integer programming for container stowage problem. In: Proceedings of the Int. Conference on Computational Science, Part I. pp. 853-862. Springer (2008), LNCS 5101

20. Nugroho, S.: Case-based stowage planning for container ships. In: The Int. Logistics Congress (2004)

21. Pacino, D., Jensen, R.M.: A local search extended placement heuristic for stowing under deck bays of container vessels. In: The 4th Int. Workshop on Freight Transportation and Logistics (ODYSSEUS-09) (2009)

22. Sciomachen, A., Tanfani, A.: The master bay plan problem: a solution method based on its connection to the three-dimensional bin packing problem. IMA Journal of Management Mathematics 14, 251-269 (2003)

23. Shields, J.J.: Container stowage: A computer aided pre-planning system. Marine Technology 21(4) (1984)

24. Tupper, E.C.: Introdution to Naval Architecture. Elsevier (2009)

25. Webster, W.C., Van Dyke, P.: Container loading. a container allocation model: I introduction background, II - strategy, conclusions. In: Proceedings of ComputerAided Ship Design Engineering Summer Conference. University of Michigan (1970)

26. Wilson, I.D., P., R.: Principles of combinatorial optimization applied to containership stowage planning. Journal of Heuristics (5), 403-418 (1999)

27. Yoke, M., Low, H., Xiao, X., Liu, F., Huang, S.Y., Hsu, W.J., Li, Z.: An automated stowage planning system for large containerships. In: In Proceedings of the 4th Virtual Int. Conference on Intelligent Production Machines and Systems (2009)

28. Zhang, W.Y., Lin, Y., Ji, Z.S.: Model and algorithm for container ship stowage planning based on bin-packing problem. Journal of Marine Science and Application 4(3) (2005) 